\title{
Students' Images of Two-Variable Functions and Their Graphs
}

\author{
Eric Weber \\ Oregon State University \\ College of Education \\ Patrick W. Thompson \\ School of Mathematical and Statistical Sciences \\ Arizona State University
}

\begin{abstract}
Author Note
Eric Weber, College of Education, Oregon State University, Furman Hall 204, Corvallis, OR 97333. E-Mail: Eric.Weber@oregonstate.edu Phone: 541-737-1305

Patrick W. Thompson, School of Mathematical and Statistical Sciences, Arizona State University, P.O.Box 871804 Tempe, AZ 85287-1804. Email: pat@pat-thompson.net This research was supported by NSF Grant No. MSP-1050595. Any recommendations or conclusions stated here are the authors and do not necessarily reflect official positions of the NSF. Any recommendations or conclusions stated here are the authors and do not necessarily reflect official positions of the NSF.
\end{abstract}




\begin{abstract}
This paper presents a conceptual analysis for students' images of graphs and their extension to graphs of two-variable functions. We use the conceptual analysis, based on quantitative and covariational reasoning, to construct a hypothetical learning trajectory for how students might generalize their understanding of graphs of one variable functions to graphs of two variable functions. To evaluate the viability of this learning trajectory, we use data from two teaching experiments based on tasks intended to support development of the schemes in the HLT. We focus on the schemes that two students developed in these teaching experiments and discuss their relationship to the original HLT. We close by considering the role of covariational reasoning in generalization, consider other ways in which students might come to conceptualize graphs of two-variable functions, and discuss implications for instruction.
\end{abstract}

Keywords: two-variable functions, three dimensions, calculus, quantitative reasoning, covariational reasoning 


\section{Introduction}

Many scientific fields use functions to model and predict phenomena by identifying quantities of interest and representing those relationships graphically. These relationships often involve multiple quantities, but there is limited work that explores how mathematics and science students reason about relationships between multiple quantities and their representations, such as graphs and tables (Montiel, Vidakovic, \& Kabael, 2008; Montiel, Wilhelmi, Vidakovic, \& Elstak, 2009; Trigueros \& Martinez-Planell, 2010; Weber \& Dorko, in press). Given the prevalence of ideas in science and mathematics that require representing multiple relationships using functions and their representations simultaneously, it is important to understand how students think about generating and interpreting representations of functions, particularly graphs of functions of more than one variable. This article builds on an image of a function's graph as representation of covariation — an image that entails an invariant relationship among two or more quantities' magnitudes as they vary. We use this notion of a function's graph to construct a hypothetical learning trajectory for how students might generalize their understanding of graphs of one variable functions to graphs of two variable functions. To evaluate the viability of this learning trajectory, we use data from two teaching experiments based on tasks intended to support development of the schemes ${ }^{1}$ in the HLT.

\section{Background}

Though the process by which students learn about representing two variable functions has not been fully explored, researchers have characterized the representations students construct as they reason about tasks involving functions of two variables. For example, Yerushalmy (1997)

\footnotetext{
${ }^{1}$ We use the term 'schemes' to mean well-coordinated, complex structures of individual schema. See Thompson (2013) for expanded discussion of the term.
} 
showed that it is important to understand what is being generalized as students move from one to two variable functions using graphical and tabular representations of those functions. She studied six seventh grade algebra students in the context of describing, solving and generalizing about functions. She identified three key parts of the students' understanding of two variable functions: Identification of quantities and variation, generalizing graphical representation of a function in three dimensions, and manipulation of the algebraic conventions for defining a function. Yerushalmy found it essential that the students constructed the quantities under consideration, identified invariant relationships between the quantities, and imagined representing the invariant relationship using a symbolic function definition in both the one and two variable case. The findings of this study also suggest that it was quantities and their relationships that provided a way for students to think about each representation (graphical, tabular, algebraic) as a representation of a function. Yerushalmy's findings suggest that quantitative reasoning is crucial to students' construction of a two-variable function (and its representations), but Yerushalmy did not define quantitative reasoning as the primary focus of her work.

As another example, Trigueros and Martinez-Planell (2010) proposed and then refined a genetic decomposition of the understandings a student needs to conceive of two-variable functions and their graphs. Subsequently, Martinez-Planell and Trigueros (2012) proposed a genetic decomposition to postulate about constructions the students might make as they think about two variable functions and their graphs. The genetic decomposition consisted of the following: 
A schema of intuitive three-dimensional space, which consists of a construction of the external material world; a schema of Cartesian plane which includes the concept of points as objects and relations between variables, such as curves, functions, and regions as processes resulting from the generalization of the action of representing their component points; a schema for real numbers which includes the concept of number as an object, and arithmetic and algebraic transformations as processes, a schema for sets; a schema for real function of one real variable including function as process, operations with functions, and coordination of their different representations. (Martinez-Planell \& Trigueros, 2012, p. 368).

The authors argued that the elements of the genetic decomposition were necessary for a student to understand the "general" notion of function and its representations. However, they did not specifically propose how these ways of thinking might develop or how it might help students envision a graph of a two-variable function. Indeed, an inability to envision how a graph is generated is one of the primary difficulties that students exhibited in the excerpts that Trigueros and Martinez-Planell shared. For instance, they focused on students' abilities to conceptualize the domain of a 2-variable function and to map elements in the domain to values in the range in order to interpret a graph of a 2-variable function. However, that they did not address mental processes by which students might envision the graph of a 2 -variable function before seeing it.

Taken together, these two studies suggest ways in which students might conceive of graphs of functions of two-variables in situations where there are at least three variables that vary. However, the articles only hint at the schemes (see footnote 1) that can support students in thinking about graphs of functions of two variables, and they do not provide a complete picture of how students might develop the ways of thinking they proposed. However, our interpretation of their results support that quantitative reasoning may be a key component in how students conceive of two-variable functions. Based on these hypotheses, the major research question driving this study was: 
How do students' understandings of graphs of single-variable functions influence their generalizations to graphs of two-variable functions and what is the role of covariational reasoning in this generalization?

To make our research question sensible we must first say what we mean by “understandings of single-variable functions' graphs" and generalizations that students might make from them. In the following two sections, we describe how quantitative and covariational reasoning prepare students to think about graphs of functions and build on these ideas to offer a conceptual analysis that describes understandings of graphs of single-variable functions that we believed would support students' in developing powerful understandings of two-variable functions' graphs were they to have them. The conceptual analysis was the basis for the teaching experiment reported later in the manuscript.

\section{How Quantitative and Covariational Reasoning Prepare Students to Reason about Graphs}

Our reading of previous research on student thinking about functions suggests one of the most difficult aspects about understanding a graph of a function may be thinking about quantities and analyzing how those quantities vary in tandem (covary). Our hypothesis was that this difficulty extends to students' attempts to reason about graphs of two-variable function as well. Here, we articulate in what ways quantitative and covariational reasoning prepare students to reason about graphs of functions.

\section{Quantitative Reasoning}

Quantitative reasoning refers to a way of thinking that emphasizes a student's cognitive development of conceptual objects with which she reasons about specific mathematical situations (Smith III \& Thompson, 2008). A quantity, in Thompson’s (1994) description is “a conceptual entity. They exist in people's conceptions of situations. A person is thinking of a 
quantity when he or she conceives a quality of an object in such a way that this conception entails the quality's measurability.” (Thompson, 1994, p. 184). Thompson (2011) defined quantification as the process of conceptualizing an object and an attribute of the object so the attribute has a unit of measure. To assign values to attributes of an object, a student must have already constructed the attributes of a situation that he imagines having measures. A value then, is the numerical result of quantification of a constructed quantity.

Thus, for a student to imagine that a graph of a two-variable function is a representation of the relationship among three quantities, the student must construct those quantities, whether it is from an applied context or an abstract mathematical expression. Thus, a two-variable function's graph requires that the student conceives of at least three quantities, two or more of which determine the third using a quantitative operation. Of course, it is nearly impossible for a novice student to conceive simultaneously of a relationship between three quantities. Instead, a student can think about how quantity 1 varies with quantity 2 (with quantity 3 fixed), how quantity 2 varies with quantity 3 (with quantity 1 fixed) and how quantity 1 varies with quantity 3 (with quantity 2 fixed). This allows the student to extend a notion of a relationship between two quantities to think about more complex systems. These conceptions about quantity are the basis for the student to use covariational reasoning.

\section{Covariational Reasoning}

Saldanha and Thompson (1998) described covariational reasoning as one understanding that if two quantities changed in tandem, if either quantity has different values at different instances, it changed from one to another by assuming all intermediate values (Saldanha \& Thompson, p. 2). Thompson (2011) proposed continuous covariation as a student's construction 
of quantities and their variation that supports an image of those quantities covarying over conceptual time.

Variation, in Thompson's description, results from a student anticipating that a quantity has different values at different moments in time, which could be represented as $x=x(\mathrm{t})$. This variation always occurs over a domain of conceptual time, $(t, t+\varepsilon)$, so that the domain of conceptual time can be represented as $D=\bigcup(t, t+\varepsilon)$ (Thompson, 2011, p. 47). This characterization of variation allows the student to anticipate that the domain of conceptual time is "covered" with these intervals, so the student can imagine that the quantity varies in chunks of conceptual time while understanding that completed chunks can be thought about as the quantity having varied continuously. Thompson's characterization of variation extends to imagining two quantities covarying, represented as $\left(x_{\varepsilon}, y_{\varepsilon}\right)=\left(x\left(t_{\varepsilon}\right), y\left(t_{\varepsilon}\right)\right)$ where $\left(x_{\varepsilon}, y_{\varepsilon}\right)$ represents an image of uniting two quantities, and then varying them in tandem over intervals of conceptual time (Thompson, 2011, p. 48). If a student has this conception of covariation in mind, it is reasonable to assume they can unite a function's independent and dependent quantity into an object. As that object varies over conceptual time, the function is generated using covariation (as we describe in the subsequent HLT). Once a student is able to think about functions ${ }^{2}$ in this way, they are prepared to think about a graphical representation of that function.

\section{Hypothetical Learning Trajectory for Graphs of Two-Variable Functions}

The following sequence builds upon the description of quantitative and covariational reasoning in the previous section to describe a particular understanding of a graph for $y=f(\mathrm{x})$ and how that way of thinking extends to graphs of two-variable functions.

\footnotetext{
${ }^{2}$ Here our description of covariation assumes functions that are continuous over the real numbers.
} 
-Imagine that there are two quantities in a situation, and that $x$ and $y$ represent their values.

-Imagine that the $x$-axis represents all the possible values of a quantity's attribute measured in some unit.

-Imagine that the $y$-axis represents all the possible values of another quantity's attribute measured in some unit.

-Imagine the point that is $y$-units perpendicularly above the value of $x$ (represented on the $x$-axis). The coordinates of this point represent the values of the two quantities simultaneously.

- Imagine a graph in two dimensions as the tracing out of that point which simultaneously represents the value of $x$ and $y$ (Figure 1)
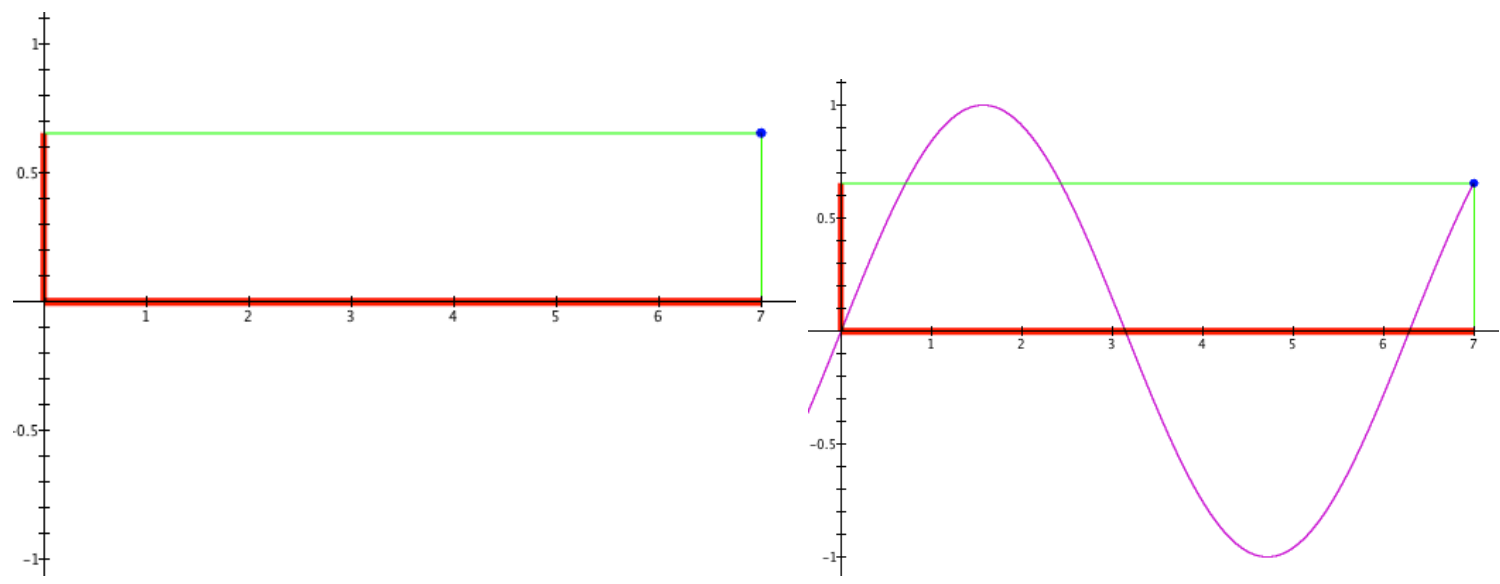

Figure 1. A point whose coordinates are the values of $x$ and $f(\mathrm{x})$ traces out $f(\mathrm{x})=\sin (\mathrm{x})$.

Building a scheme for a graph of a function that relies on covariation allows a student to think about the construction of a function's graph in two dimensions, but also allows them to think about a graph as representing completed covariation of variables. The reversibility of this scheme is crucial to the students' ability to use this scheme to understand graphs of two-variable 
functions. By reversibility, we mean that the student's covariation scheme allows them to imagine the construction of the graph by a tracing out (i.e. from symbolic form to graph) and to imagine that a given graph was generated by a tracing out (i.e. imagining a graph was created by a point tracing out according to a relationship between quantities defined in symbolic form). This reversibility is key to students' construction and interpretation of surfaces in space.

\section{Extension to Graphs in Three Dimensions}

The idea of covariation can be considered from the perspective of parametric functions, where thinking about $(\mathrm{x}, \mathrm{y})=(\mathrm{x}(\mathrm{t}), \mathrm{y}(\mathrm{t}))=(\mathrm{t}, \mathrm{f}(\mathrm{t}))$ supports an image of scanning through values of one variable and tracking the value of another variable. This image supports a student imagining the quantities as coupled. This way of thinking could support reasoning about a function defined parametrically, such as $(x, y)=(\sin 10 t, \cos 20 t), 0 \leq t \leq 1$. Thompson used this example to suggest a way of thinking about curves in space, such as $(x, y, z)=(\sin 10 t, \cos 20 t, t), 0 \leq t \leq 1$, by imagining that $t$ is actually an axis, "coming straight at your eyes" (Oehrtman, Carlson, \& Thompson, 2008). Thompson proposed that this way of thinking about a curve in space could help the student visualize the graph of the function defined by $z=f(\mathrm{x}, \mathrm{y})$, by thinking about $y$ or $x$ as a parameter.

-Imagine the function $f$, defined $f(\mathrm{x})=a \sin (\mathrm{x})$, where $a$ is a parameter value. Think about the graph of $f$ as if it is the graph of $f$, defined $\mathrm{f}(\mathrm{x})=a \sin (\mathrm{x})$, but drawn $a$ units perpendicularly from the sheet of paper on which it seems to appear (that is, think of the sheet of paper is actually a glass plane. The graph is behind the paper when $a$ is negative, and it is in front of the paper when $a$ is positive.

-Imagine $a$ as an axis perpendicular to the flat sheet of paper at the $x-y$ origin, and imagine starting with a negative value of $a$, say $f(\mathrm{x})=-2 \sin \mathrm{x}$, pull the graph of the function 
$f(\mathrm{x})$ from behind the paper toward you. As you imagine pulling the graph toward you, imagine that the graph adjusts for the changing value of $a$ while also imagining its distance from the $x-y$ plane being $a$ units behind or in front of it.

-Imagine that as you pull the function's graph along the $a$ axis, the graph of $f(x)=a \sin (x)$ has "fairy dust" on it, which creates a thin surface as the graph of $f$ is pulled along the $a$ axis. Each point on this surface has three coordinates: $x, a$, and $f(x)$.

The net effect of this collective of coordinated images and meanings is that one sees a surface being generated as $x$ varies to produce the graph of $f$ for a particular value of $a$ while the value of $a$ varies to sweep out a surface generated by the family of graphs generated by $x, f$, and $a$ (Figure 2). Thus, when students see a surface in space, they imagine it represents the result of tracking a relationship between quantities having covaried.
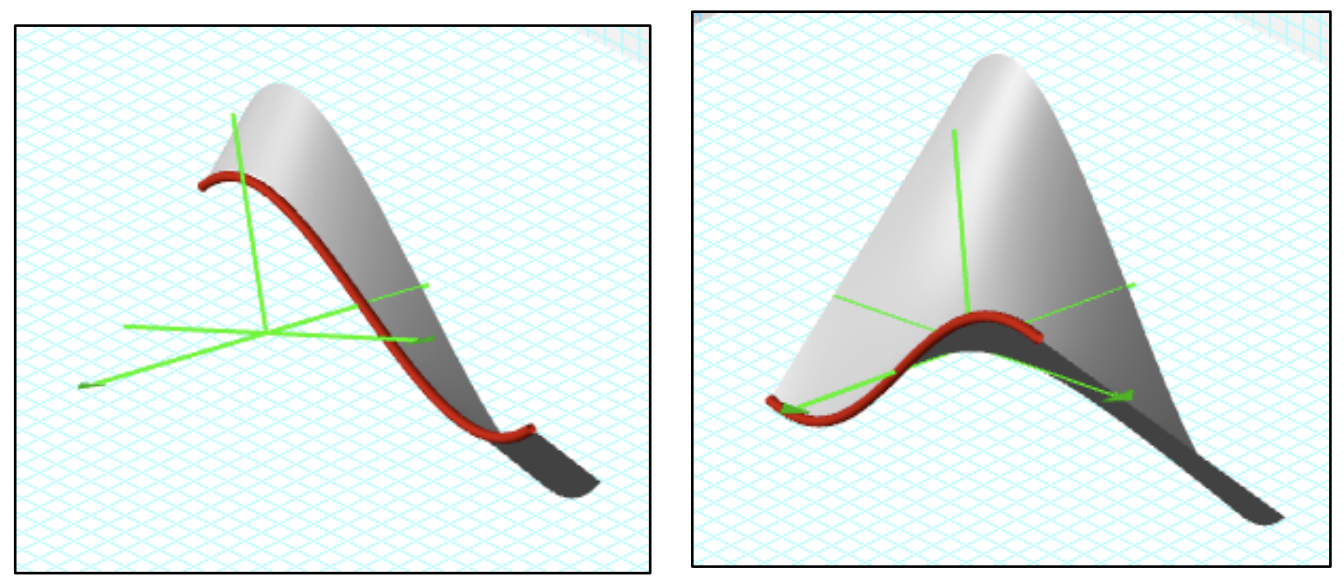

Figure 2. Tracing out the graph of the function $f$, where $f(x, y)=y \sin (x)$.

It is important to note that this scheme does not account for how a student might think about graphs of every single or two-variable function. Instead, this scheme for graph as covariation allows a student learning about functions of two-variables to meaningfully extend an understanding of graphs of single variable functions. It is also important to note that while we focus on examples of two variable functions in this paper that can be conceived of as $g(\mathrm{y}) h(\mathrm{x})$, 
such as $f(\mathrm{x}, \mathrm{y})=x y$, we do so to help students more easily assimilate the family of planar graphs. The idea of two stages of sweeping out as described in the conceptual analysis are much more general than this, and apply to functions beyond those that can be written as $g(\mathrm{y}) h(\mathrm{x})$. Together, these schemes are intended to propose a way in which students might come to think about graphs of two-variable functions by generalizing their schemes for graphs of single variable functions. The following section describes tasks that we designed to support the development of these schemes.

\section{Task Sequence for Graphs of Two-Variable Functions}

In this section, we describe activities that we used in a teaching experiment to evaluate the viability of the schemes described earlier. Together, the scheme and the activities intended to engender it represent a hypothetical learning trajectory for graphs of two-variable functions.

\section{The Difference Function}

We use this activity to help students think about polynomial functions as sums of monomial functions (Dugdale, Wagner, \& Kibbey, 1992) with an explicit focus on quantitative reasoning. We also use this task to gain insight into how quantitative reasoning, or lack thereof, could support or constrain an image of the polynomial function as dependent on its monomial parts. Students are asked to graph the functions $f$ and $g$ by describing how $x$ and $f(\mathrm{x})$ and $g(\mathrm{x})$ covary.

- $\quad$ Construct the graph and explain the behavior of the functions $f(x)=x^{2}$ and $g(\mathrm{x})=2 \mathrm{x}$.

- $\quad$ As $x$ increases from 0 to 1 , why does $f(x)=x^{2}$ behave in the way you have indicated?

- $\quad$ As $x$ increases how do $f(\mathrm{x})$ and $g(\mathrm{x})$ vary?

- $\quad$ Construct a graph of $h(x)=x^{2}-2 x$, and explain why it behaves in the way it does based on their descriptions of the behavior of $f(\mathrm{x})$ and $g(\mathrm{x})$.

We then use the activity to support the student in describing the behavior of $h(\mathrm{x})$ based on tracking a difference between $f$ and $g$, and creating the graph as a representation of that 
difference. The intention is students come to see every point on the graphs of $f(\mathrm{x})$ and $g(\mathrm{x})$ as a representation of the relationship between $x$ and the respective function, and every point on the graph of $h(\mathrm{x})$ as a representation of the relationship between $x$ and the difference of $f(\mathrm{x})$ and $g(\mathrm{x})^{3}$. This image supports students' development of an image of a graph in two dimensions as comprised of an infinite number of points whose coordinates depend on quantities' values.

\section{Sweeping Out as Means for Constructing a Surface}

We use an extension of the difference function task with the goal of helping the student develop an image of $a$ as a third axis along which a function's graph represented in a plane sweeps out to generate a surface in space. The students are asked to describe the effect of a parameter, $a$, on the graph of the function $h$ for the graph of a new function $m$, defined $m(\mathrm{x})=$ $a \mathrm{~h}(\mathrm{x})$. The intention is that students conceive of $a$ having two effects. First, $a$ shifts every point on the graph of the function $h$, and $a$ causes that graph to move along a third axis perpendicular to the graph of the function $h$. When the students imagine tracing out a trail of the graph as $a$ changes, they can begin to visualize a surface being generated in space.

The intention of this activity is that students develop an image of a graph in both two and three dimensions as the result tracing the path of a sweeping out (a point, and a cross section, respectively), and anticipate that a given graph is a representation of that sweeping out having occurred. It is also important that students begin to think about sweeping out occurring along multiple axes because a key component of the scheme for graphs is that the generated surface is invariant even with the choice of sweeping out along the $x, y$ and $z$ axes.

\section{Construction and Interpretation of a Surface}

\footnotetext{
${ }^{3}$ Note that the notion of a difference function applies widely, not just to polynomial functions. We focus on polynomial functions in this activity because of their familiarity to students.
} 
In part one of this activity, students develop a process for constructing the graph of a twovariable function $\left(f(x, y)=x^{2} y^{2}\right)$ given its algebraic definition. Part one moves students from using parameterization explicitly to introducing parameterization as a way to conceive of a given two-variable function's graph. That is why it is important students see sweeping out as invariant, so that they can imagine parameterizing any of the given variables and imagining the sweeping out occurring along the axis that was parameterized to generate the surface in space.

In part two, students define a function that could represent a given graph of a twovariable function (Figure 3). Meaningful responses to this task rely on students' use of their image for a graph as a representation of variables having covaried. They begin to think about coordinating the sweeping out having occurred in multiple planes, determine what function could be represented in the $\mathrm{z}-\mathrm{x}, \mathrm{z}-\mathrm{y}$ and $\mathrm{x}-\mathrm{y}$ cross sections, and attempt to coordinate those cross sections into the graph of a two-variable function.

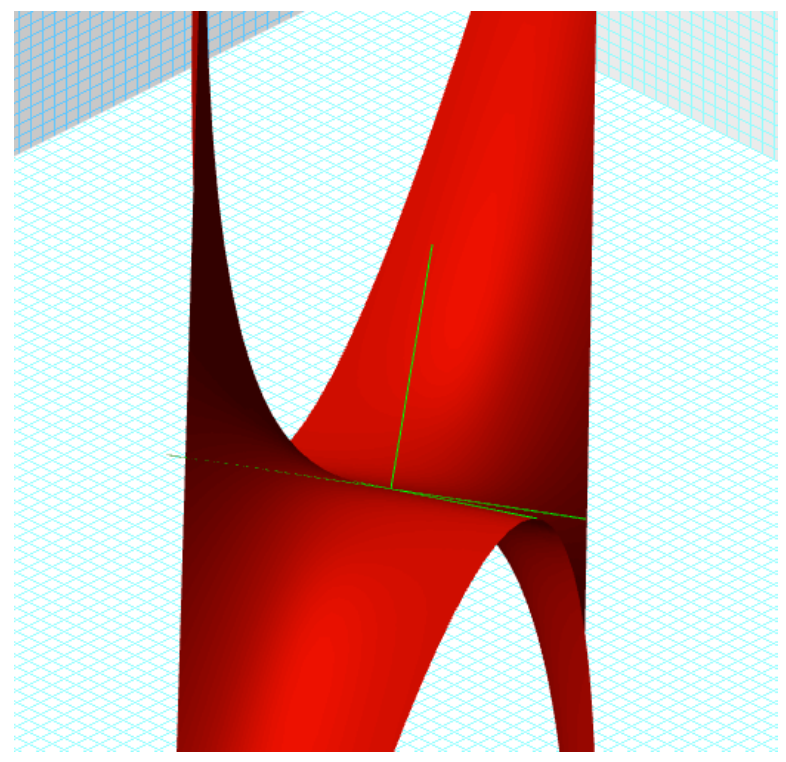

Figure 3: Surface displayed to students. 
The above tasks are a hypothesized sequence by which we anticipated students might come to develop the schemes we described in the conceptual analysis of graphs of functions of two-variables. Thus, prior to the teaching experiment we believed that this hypothetical learning trajectory could support a student in thinking about a graph as the representation variables having covaried. In the following sections, we report on the teaching experiment intended to evaluate the viability of our hypotheses.

\section{Method}

\section{Subjects and Setting}

Two students, Jesse and Lana, were in the process of taking a single-variable calculus course, in which the first author was a non-participant observer and the second author was the instructor. Lana was a first year college student, and Jesse was returning to college as a nontraditional student. They were selected because of their ability to describe the images they had for mathematical ideas as well as their willingness and availability to participate in intense instruction over the course of three weeks. The course was grounded in quantitative and covariational reasoning but none of students had previously seen a function of more than one variable. During all of the teaching sessions, the students were able to use a laptop computer with a Graphing Calculator program. Each session was videotaped and the laptop screen animations were recorded. The laptop computer was used to help students represent ideas in three dimensions that they were attempting to sketch on paper or as a support to help them think about sweeping a plane through three dimensions. The students were able to use the computer whenever they felt compelled, but also were asked to explain for what reason they felt they needed to do so. Thus, the computer was not used as a "teaching tool", but as a means of support controlled by the student. 


\section{Use of Graphing Calculator}

The students were encouraged to use the Graphing Calculator, a computer graphing program created by Ron Avitzur, (GC, Avitzur, 2011), whenever they wished to represent or illustrate their thinking during the teaching sessions. We found that the students typically used GC to confirm or disconfirm what they already appeared to be visualizing. In most cases, the students' creation of graphs in GC fit their schemes. For instance, Lana often used GC to type a function definition to see what graph was generated to "file away in her memory". Jesse used GC in an attempt to program the covariation he anticipated. We do not think that their ability to use GC necessarily allowed them to develop a way of thinking or visualization that they otherwise could not. However, we do think the ability to confirm their hypotheses using GC allowed them to be more certain about their ideas, as we illustrate in the analysis section.

\section{Teaching Experiments and Reflexivity}

The teaching experiment sessions focused on creating models of student thinking while we attempted to help them think in sophisticated ways about graphs of functions. We built on Steffe and Thompson's (2000) model for a teaching experiment and accounted for our role in what the student said and did and understand that our interpretations of student's behavior and explanations contributed to the students' actions, because our actions toward them were predicated on our understandings of what they did. We reflected on how students interpreted our actions and how that interpretation might have played a role in the students' actions. Immediately after each teaching experiment session, we watched the recording of the session, did a basic transcription, and reflected on how the student's actions within that session necessitated adjustments or changes in the models of student thinking. 


\section{Analytical Method}

Thompson (2008) described one use of conceptual analysis as to propose models of student thinking that help to make distinctions in students' ways and means of operating, and to explain how these ways of and means of operating persisted or changed during the teaching experiment. Our retrospective analyses involved making interpretations and hypotheses about students' thinking that we did not have in the moment of the teaching experiment. As we made interpretations and hypotheses about the students' understandings using retrospective analysis, we used conceptual analysis to continually generate and revise a scheme of meanings that would have made what the students said and did coherent for them. Using retrospective and conceptual analysis required developing a systematic coding scheme for the data. The coding process centered on theorizing about ways of thinking that explained categories of student behavior by using a combination of open and axial coding (Strauss \& Corbin, 1998). The coding of video data served two purposes. It was a way to create objective counts of various coded instances. Second, the use of Studiocode to code video instances allowed us to generate videos that contain all instances falling under a particular code, which supported further conceptual analysis of ways of thinking that were allied with the behavior that the code marks.

\section{Jesse's Scheme for Graphs}

Our initial interviews with Jesse (prior to the teaching experiment) suggested that he conceived of a function as a "rule" between two variables, and that a graph of the function "visually displayed that rule for every point." These two comments by Jesse appeared repeatedly in our initial discussions, suggesting the he conceived of a function as an invariant relationship between variables, and that a graph of a function adhered to that invariant relationship for every point comprising it. We think these initial ways of thinking about a function and its graph 
supported Jesse in developing more sophisticated notions of covariation in the teaching experiment itself.

The first excerpt comes after Jesse had created several graphs of $y=f(x)$, where $f(x)=a\left(x^{2}-2 x\right)$, that corresponded to different values of $a$ (Figure 4). We were interested to see whether Jesse could think about $a$ as a parameter and its effect on $f$ 's graph, and then conceptualize $a$ as another variable represented on a third axis that comes straight out of the screen. We conjectured that this imagery would support Jesse's use of his covariation scheme to conceive of $a$ as varying while sliding $f$ 's graph through space, adjusting $f$ 's graph as he imagined the value of $a$ varying.

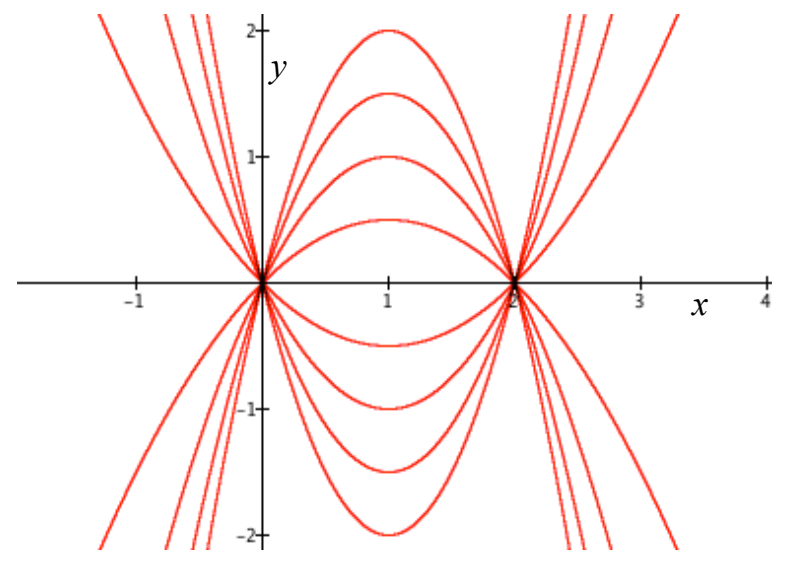

Figure 4. Jesse's graphs of $y=f(x)$, where $f(x)=a\left(x^{2}-2 x\right)$ for various values of $a$.

Excerpt 1 is Jesse's response to the interviewer's request to imagine that values of $a$ are on an axis that is perpendicular to the computer screen.

\section{Excerpt 1. Jesse describes the effect of a. J: Jesse, I: Interviewer}

1 I: Let's say a represents the distance of the graph in front of or in back of the laptop

2 screen. Can you anticipate how the graph will behave as we change the value of $a$ ?

$3 \mathrm{~J}$ : (Pause.) I kind of think the parameter changing would fill in a shape, like a

4 windshield wiper sort of, and it would cloud over because the function is stretching

$5 \quad$ vertically in both directions as the value of $a$ changes.

6 I: Could you say a little more about what you are imagining?

$7 \mathrm{~J}$ : Yeah, well, this function is going to sweep up and down (Figure 4), and after awhile,

8 will fill in the window I am seeing. Weird! It's like I have to consider depth. 
9 I: Consider depth?

$10 \mathrm{~J}$ : Well, the parameter is affecting the behavior of the points on the graph, but I am

11 having to remember that when the parameter changes, like we said, the graph is

12 moving toward and away from me as well. So things are changing in a number of

13 places because of the parameter.

14 I: Could you draw what you are seeing?

15 J: (See Figure 5)

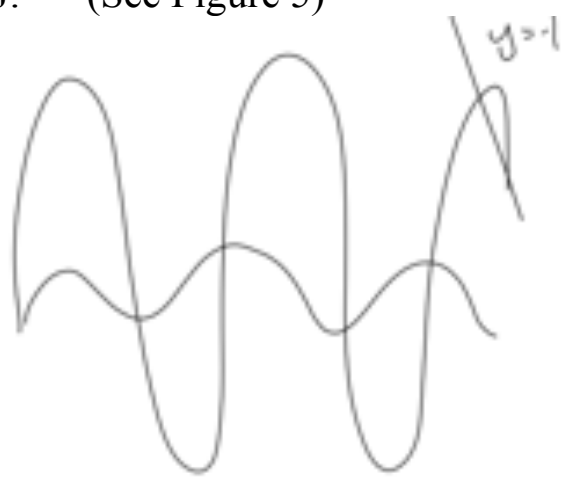

Figure 5. Jesse's drawing to show the graph of the function for two values of $a$.

Jesse noted two effects of changing the value of $a$. First, the graph underwent vertical stretching by affecting many points on the graph (Excerpt 1, Lines 4-5). Second, the graph moved along the $a$-axis (Excerpt 1, Lines 11-13). His work in Figure 5 illustrates the function's graph for two values of $a$. Jesse anticipated that iterating through values $a$ would simultaneously move the graph up and down, producing a windshield wiper effect. He coordinated this windshield wiper effect with the graph moving toward and away from him (Excerpt 1, Line 12). Taken together, these images became his basis for describing a sweeping out of a graph in the plane through space.

Excerpt 2 (below), follows Jesse's invention of what he called the " $z-x$ " and " $z-y$ " perspectives. The $z$-x perspective, for example, was to imagine varying a graph of $z=f(x)$ along the $y$-axis. Jesse anticipated that he could imagine the surface generated by a function of two variables by using his perspective scheme for $z-x, z-y$ and $x-y$ perspectives. Jesse's scheme for the construction of a graph in space relied on coordinating three images, 1) the perspective from which he would sweep out the surface, 2) the graph that would be swept out (the generating 
graph), and 3) tracing the generating function's behavior for all values of a parameter in an interval (treated like a variable).

Excerpt 2. Jesse anticipates the graph of a two-variable function.

1 I: Suppose I have the function $f(x, y)=x^{2} y^{2}$ could you talk about how you think $2 \quad$ about it?

3 J: I know by how it is defined that two variables vary in different directions... Going 4 back to perspectives, hmm, let me calculate this here... I would look at the $\mathrm{z}$-X and $5 \quad z-y$ perspective, and here you would have a parameter times $y$ squared in $z-x$ and a 6 parameter times $x$ squared in $z-y$, so you would see parabolas from each 7 perspective...

8 I: If you did that for this function, what do you think you would see from the $x-y$ 9 perspective?

$10 \mathrm{~J}$ : Well, it would be a function like $f(x)=a / x^{2}$, so if I graph that would produce a 11 cross section that sweeps out to produce the same graph.

It is important to note that Jesse's description of $f(x)=a / x^{2}$ does not reflect the more accurate $\mathrm{f}(x)=a / x$. This suggests that the notion of parameterization did not immediately extend to all three variables. Indeed, as Jesse built the idea of perspective, which meant that he could generate a graph by sweeping a $y$-line along the $x$-axis, or by sweeping an $x$-line along the $y$-axis ( $\mathrm{z}-\mathrm{x}$ and $\mathrm{z}-\mathrm{y}$ perspectives) he sometimes had difficulty thinking about the $\mathrm{x}-\mathrm{y}$ perspective in which $z$ was parameterized. This issue brings up a natural limitation of the sweeping out process because in some cases parameterization of $z$ makes the object cease to be a function.

\section{Excerpt 3. Jesse describes his understanding of a graph in three dimensions.}

1 I: How does the sweeping out idea come into play when we are looking at a general $2 \quad$ surface in three dimensions?

$3 \mathrm{~J}$ : I imagine that sweeping out happening from multiple ways. I kind of created the $z-x$

$4 \quad$ view before, where $a$ was like the $y$-variable. I could look at the $z-y$ view too, where $5 x$ is the parameter and $y$ is the variable. This would be like a $z-y$ view. These are

$6 \quad$ like perspectives.

7 I: What are some things you associate with a given surface?

8 J: I would first focus on its shape, to see if it fits some prescribed things I know, but 9 then I would shift to how it was made. I could do a sweeping out any perspective.

10 So, let me say this, the sweeping out starts in the plane, so a point sweeps out, then 11 that entire thing sweeps out along another axis. This could happen in any way, $\mathrm{z}-\mathrm{X}$ 12 or z-y. 
Three statements by Jesse stand out to us. First, Jesse saw multiple levels of covariation happening: sweeping out a point in a plane, and then sweeping out the object created by that point through space to produce a surface. Second, Jesse said that he would look to see if a given graph is familiar, but even if so he would "shift to see how it was made" (Excerpt 3, Line 9). This to us indicates strongly that Jesse anticipated that graphs emerged through variables' covariation. Third, Jesse anticipated that each sweeping out (regardless of perspective) would generate the same surface in space (Excerpt 3, Lines 11-12), so that Jesse anticipated that graphs are invariant across perspectives.

Jesse's scheme for constructing a graph in space appeared to also be reversible as suggested by his ability to determine the algebraic definition of a given graph (Figure 3). Excerpt 4. Jesse determines a function that will have a given surface as its graph.

1 I: So, let's take a look at this surface that I generated. Could you describe a function 2 that it could represent? Algebraic definition, I mean.

$3 \mathrm{~J}$ : Hmm, this is pretty hard, more thinking backwards type of stuff. I guess I would 4 have to decide which perspective I am looking at. So if this is z-y, it looks like it 5 could have been generated by a moving parabola. Then in z-x, well, it looks linear 6 in some places, so maybe produced by a moving linear function.

7 I: So, based on what you have said, how might you define the function?

$8 \mathrm{~J}$ : My best guess is like $a y^{2}$ from z-y and $b x$ from z-x, so a parameter times $x y^{2}$

Jesse's approach to determining a function that would have Figure 3 as its graph entailed (1) selecting a perspective from which to look (Excerpt 4, Line 4), (2) visualizing the cross sections from a that perspective (Excerpt 4, Line 5), and (3) determining an algebraic definition of a function that could represent that cross section (Excerpt 4, Line 8). In Excerpt 4, Jesse applied this scheme twice, once from each of the $\mathrm{z}-\mathrm{x}$ and $\mathrm{z}-\mathrm{y}$ perspectives, and coordinated those applications to determine that $z=a x y^{2}$ could represent the given surface. 
We believe that Jesse saw the graph of a two-variable function as a result of three variables' continuous variation. His use of perspective and generating functions appeared to be a way to parse the representation of three covarying variables into coordinating graphs of two covarying variables. As a result, Jesse's schemes for the construction and interpretation of a twovariable function's graph relied on his image of what the graph represented (covariation) and how that graph was generated (sweeping out).

\section{Lana's Scheme for Graphs}

Our initial interviews with Lana (prior to the teaching experiment) suggested that she conceived of a function as "two variables connected by an equals sign", and knew that "for every equation I have there is a picture that goes with it, or a graph.” Thus, there was no notion of invariance between variables, or even a conception of dependence between them. He repeated use of a function as an equation and a graph as a picture associated with that equation constrained her from developing images of covariation in the way that Jesse did.

Lana's understanding of a graph was characterized by her conception of a graph as associated with an algebraic definition, rather than generated by it. We describe Lana's scheme for graphs, and articulate the difficulty we believe her scheme created for her thinking about covariation. Excerpt 5 is Lana's response to the interviewer's request to imagine that values of $a$ are on an axis that is perpendicular to the computer screen.

\section{Excerpt 5. Lana exhibits thinks about the effect of a. L: Lana, I: Interviewer}

1 L: Okay, well, as $a$ changes, the humps of this function are going to get larger as $a$ gets

2 more negative and more positive, kind of creating tunnels that open. A strip of

3 paper is kind of like my starting graph, and then adjusts when $a$ changes.

4 I: Okay, could you say a little more, where are these humps coming from, what makes

5 them appear?

6 L: The tracing out happens when you follow and track where the strip of paper has 7 been. 
8 I: Okay, so does this [fit] with your understanding of a graph?

9 L: Well, like in the case of a graph, I have a stripwire type thing that moves around

10 and molds differently. Function changes, graph changes, I think. The remolding

11 happens here when I change the value of $a$ by one at a time.

Lana anticipated that a change in the value of $a$ affected the graph, and that these changes created what she described as tunnels when tracked over values of $a$ (Excerpt 5, Lines 2-3). We interpreted her to mean that when the value of $a$ changed, a new graph appeared, where the new graph adjusted its shape to fit the new function definition (Excerpt 5, Lines 9-10). Lana's description of 'function changes, graph changes' (Excerpt 5, Lines 10-11) suggests she saw each cross-section as the result of changing the value of $a$ in the definition, but there was no indication she saw points shifting to create the new graph.

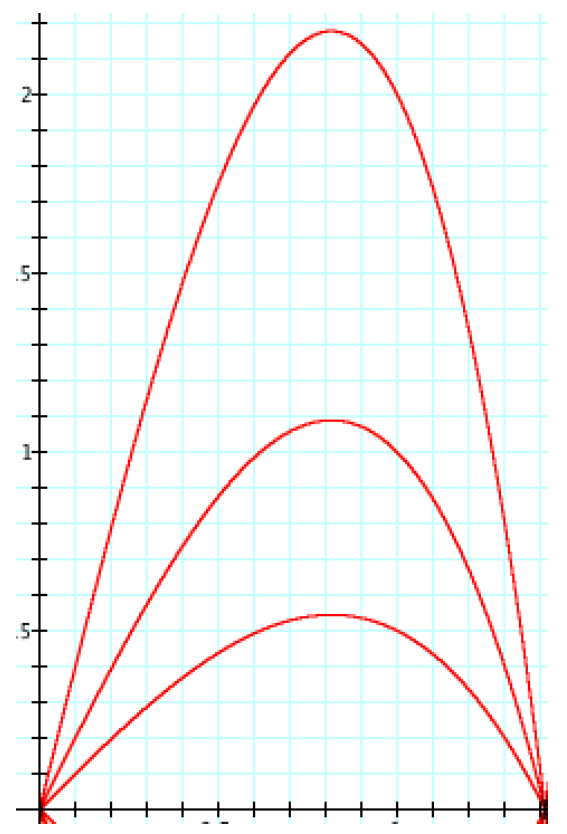

Figure 6. Lana's representation of the function $h$ for three values of $a$.

Over the next two days, with the interviewer's support, Lana developed an approach much like Jesse's perspectives. Her approach, like Jesse's, involved a graph in a plane sweeping out along an axis perpendicular to it, but her scheme did not entail an image of covariation (Excerpt 6, Lines 3-5). For instance, Lana's inability to visualize what would be swept out until 
seeing the sweeping out was completed (Excerpt 6, Lines 5-7) and her need to see the whole before seeing each part (Excerpt 6, Lines 10-12) suggest her image of sweeping out was did not fit with her image of a surface. We believe this issue arose because her scheme for graph involved matching a graph with an algebraic definition, rather than using the definition to construct perspectives to visualize how variables covary.

\section{Excerpt 6. Lana describes how she thinks about surfaces.}

1 I: You mentioned earlier the perspectives help you look at the graph in different $2 \quad$ ways?

3 L: Yeah, well, for z-x, I just treat $y$ like a parameter, and z-y, treat $x$ like a parameter. I

4 figure, I can do this and have an $\mathrm{x}-\mathrm{y}$ view too where $z$ is a parameter but that does

5 not always make sense. But I can't visualize the exact sweeping out until I graph it.

6 I mean, I know how it gets created, I think, but I can't see it until I look at it in

7 graphing calculator, either on the computer or in an actual calculator.

8 I: What about the process you just described? Does that help you think about how the $9 \quad$ graph was created?

10 L: No, not really, because I really need to get an idea of what the surface looks like,

11 its kind of been like looking at maps for me, and imagining I sort of have to see the 12 whole before I can see each view and part.

We think Lana's desire to associate a graph with its algebraic definition in turn led her to associate function definitions with shapes of graphs (Excerpt 7, Line 3). Her attribution of parabolas to a property of 'the algebra' (Excerpt 7, Line 5), further suggests that she was thinking about a function as defined by the physical features of a graph.

\section{Excerpt 7. Lana determines the behavior of a graph in space.}

1 I: Let's say I have a function $f, f(x, y)=x^{2} y^{2}$. Can you take me through how you $2 \quad$ would think about this function?

3 L: It has squares in it, which make me think parabolas right away.

4 I: Where do these parabolas come from?

5 L: The algebra says they are there I think. If you have a square, something is probably 6 a parabola.

In Excerpt 8, Lana tried to determine a function that could represent the graph in Figure 3 , but her need to match a graph with a known definition constrained her from doing so. 


\section{Excerpt 8. Lana describes matching a graph with a function definition.}

1 I: Suppose you have a graph of a surface in space, but do not know the function it

$2 \quad$ represents. How might you about hypothesizing about it?

3 L: Well, I look at the shapes I notice in it, like sort of I was to look at a map without

$4 \quad$ labels. If the map has no labels, I would look for landmark features, like bumps and

5 hills. But if I have not seen it before I would have trouble understanding what it was

6 a map of.

$7 \quad$ I: So, if the surface was not one with which you were familiar prior?

8 L: Then there is not a good chance I could figure out what function it is a graph of.

Lana's approach to determining a function that a given graph could represent entailed matching the graph with a known function, similar to matching a face with a name (Excerpt 8 , Lines 3-4). Because her scheme did not allow her to imagine the construction of the surface, she had no means to determine what function could have generated the graph. Our analyses suggest that Lana's scheme for a graph consisted of a visual object (in the plane or in space) and its defining features (e.g. slant, bumps, humps) along with a name (algebraic definition) associated with those features. Thus, for Lana, learning about functions and their graphs entailed memorization rather than attention to a process that governed the construction of a graph.

However, we do not intend to be negative with regard to Lana's conceptions of a graph. While covariational reasoning did not fit with her scheme, she was able to recognize functions by their shapes, often from an amazing array of memorized exemplars. We anticipate that other approaches in instruction may have been more effective for her. For example, a decomposition approach, which involves finding the shape of the graph by taking cross sections from $\mathrm{z}-\mathrm{x}, \mathrm{z}-\mathrm{y}$ and $x-y$ perspectives could have helped her apply her approach of memorization of shapes and their function definitions. By coordinating from these three perspectives, she may have been able to determine the function that a surface represented without relying on the covariational reasoning approach that Jesse did. Moreover, her catalogue approach to two-dimensional graphs did not constrain her from imagining the graphs sweeping out through space, and we anticipate 
she could have been led to understand the global move of these curves to generate a surface in space without relying on notions of covariation. This is to say that while Lana's approach did not rely on covariational reasoning, it is plausible that she could have developed sophisticated schemes for graphs of two-variable functions were a different task sequence or type of instruction to be used. However, because our central question relied on covariational reasoning, we did not explore this possibility in the study.

\section{Discussion}

\section{Research Question}

Recall that the major question driving this study was:

How do students' understandings of graphs of single-variable functions influence their generalizations to graphs of two-variable functions and what is the role of covariational reasoning in this generalization?

To answer this question, it is important to consider what Lana and Jesse generalized as they moved from thinking about graphs of one variable functions to thinking about graphs of two-variable functions. We think that each student generalized what his or her schemes for onevariable function's graphs would support. For example, Jesse's covariation scheme allowed him to think about a graph as composed of points that represent variables' covarying, so his generalization from single to two-variable functions consisted of describing a process to generate that covariation. In his case, that process was the sweeping out of a point in the plane to generate a graph, and sweeping a graph through space to generate a surface. In contrast, Lana's connection between graphs in two and three dimensions was her desire to name them with a function based on their shape. Thus, there was little generalization necessary when thinking about graphs of two-variable functions because she was only memorizing new graphs, not 
thinking about the rules governing their construction. Thus, while a covariation scheme is not the only way to think about graphs, Jesse's case is consistent with our claim that covariational reasoning provides a means for students to generalize their understanding of functions and graphs, and Lana's case is consistent with our claim that not thinking covariationally may obstruct such generalizations. However, we cannot say that Lana's case illustrates that her approach to functions' graphs is intractable at a global level. Instead, we believe her approach is intractable with a covariational reasoning approach.

\section{Relationship of Results to Literature on Visualization}

It might appear that the research on visualization (Krutetskii, 1969, 1976; Presmeg, 1986, 1992, 2006) is related to Lana and Jesse's ways of thinking about graphs. For instance, one could consider Jesse's thinking about graphs to be related to Krutetskii's visual reasoning construct, and Lana's thinking about graphs to be related to Krutetskii's analytical reasoning construct. One could also view Lana's thinking as related to Presmeg's construct of prototypes (Presmeg, 1986, 1992) because Lana used shapes to help her associate a face (the graph) with a name (the equation). On the other hand, the connection becomes less clear when we note that Jesse expressed his visual reasoning in symbolic notation, and Lana's interpretations of her symbolic formulations were in terms of associated shapes. We therefore do not appeal to Krutetskii's or Presmeg's visualization constructs in our interpretations of Jesse's and Lana's mathematical reasoning. Instead, we appeal to the more fine-grained and intertwined constructs of imagery and operation offered by Piaget's genetic epistemology..

\section{Relationship of Results to Other Existing Literature}

Recall that the hypotheses driving this study were based upon the previous work of Yerushalmy (1997) and Trigueros and Martinez-Planell (2010, 2012). Our interpretation of their 
results suggested to us the central role of quantitative reasoning for graphical representations and the need to propose an HLT and set of tasks by which students might come to understand graphs of two-variable functions, in contrast to describing what a sophisticated understanding might look like without presenting how it might develop. Our results support the notion that quantitative reasoning is important for students' conceiving of graphs of functions. Moreover, our study sheds light on the powerful understandings students might achieve when they are able to reason covariationally with a quantitative reasoning basis (as Yerushalmy's results suggested), as well as the issues that arise when a student cannot reason in these ways.

Our results also highlight the importance of proposing and evaluating schemes and tasks to support their development to evaluate the viability of how a student might come to understand surfaces in space. Finally, we think that our results go beyond illustrating the usefulness of covariational reasoning. In particular, the notion of parameterization and subsequent iteration of a variable to produce a family of function's graphs which can be conceived of as a sweeping out of a surface in space is an approach that merits further attention. Lana's results make us especially curious as to whether parameterization and sweeping out require covariational reasoning, and if they do not, does covariational reasoning affect how a student understands the result of the sweeping out process?

\section{Suitability of Sweeping Out Approach for Instruction}

We want to emphasize that we see the sweeping out of a planar surface as just one way to systematically generate a two-dimensional domain and a graph of a two-variable function. For instance, one can generate the two-dimensional domain with an expanding circle (pre-image of a level curve) while generating the range by tracing the path of the level curves (expanding circles). In Figure 7, the two-dimensional domain is generated by holding $z$ at zero, 
parameterizing $x$ and varying the value of $y$, which expands the circle as the value of $y$ increases (left). Simultaneously, the range is generated by considering each circle as the pre-image of a level curve and tracing the sweeping out of those level curves. The sweeping out of the level curves takes place by making $z$ a function of $y$. Then, as $y$ varies, the tracing out of the level curve generates the surface (middle).

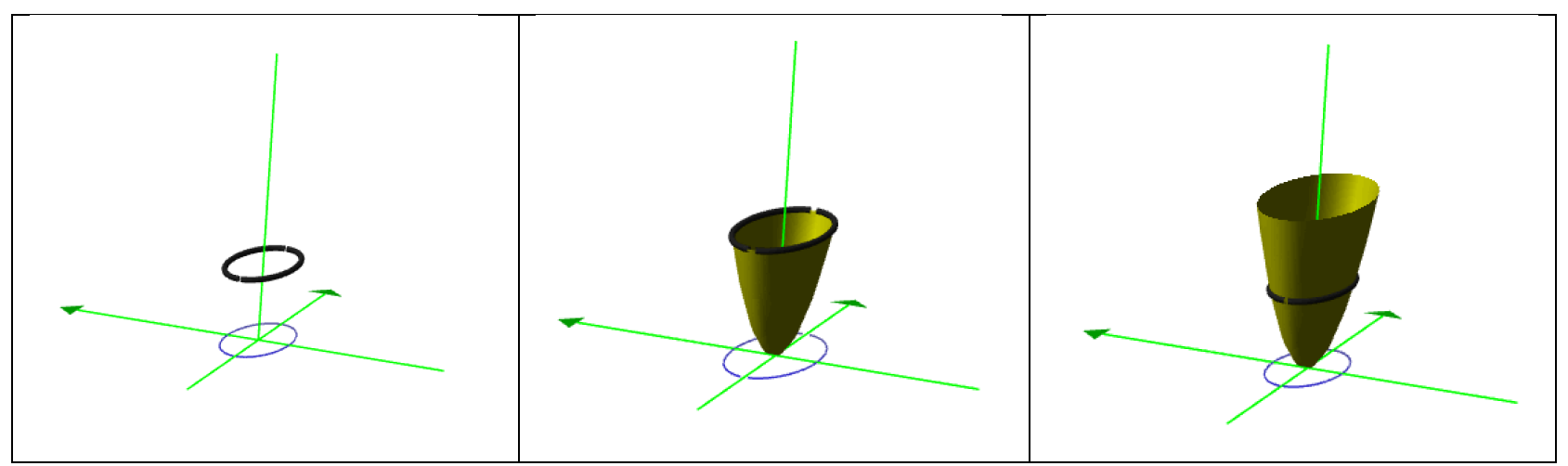

Figure 7. Expanding Circles

The various ways to generate a graph of a two-variable function bring up a larger issue: what are the issues associated with using a sweeping out scheme for graphs? For instance, it is an open question as to how well a sweeping out would be able to help students identify things like local maxima/minima, saddle points. For instance, it might be that fitting together different perspectives help provide a global visualization of maxima, minima and saddle points more than sweeping in one way. It is also unknown how this notion of generating surfaces in space might support students' development of notions of directional derivative. However, our hypothesis is that Jesse would be naturally positioned to think about rate as a quantification of how variables are covarying, while Lana would have difficulty thinking about quantities because of her association of functions with shapes. For instance, we anticipate it would be difficult for Lana to measure and interpret rate in a way that focuses on how fast quantities are varying unless she 
could conceive of a function as representing quantities, while Jesse might be naturally attuned to do so because of his attention to covariation.

Many of these unknowns result from a natural limitation of this study: that it included only two students, only one of which encountered significant difficulty during the teaching experiment. While the small number of subjects was intentional so as to provide insight into the development of their schemes for graphs, it limits the claims we can make about the applicability of the schemes we found to a general population of students learning these ideas. Future studies that address these issues would a) increase the number of students who engage in the teaching experiments, $b$ ) ensure that students with a range of abilities to think covariationally were involved in the study, and c) draw from courses in which quantitative and covariational reasoning were not the main foci. 


\section{References}

Avitzur, R. (2011). Graphing Calculator (Version 4.0). Berkeley, CA: Pacific Tech.

Dugdale, S., Wagner, L. J., \& Kibbey, D. (1992). Visualizing polynomial functions: New insights from an old method in a new medium. Journal of Computers in Mathematics and Science Teaching, 11(2), 123-142.

Krutetskii, V. A. (1969). An analysis of the individual structure of mathematical abilities in schoolchildren. Soviet studies in the psychology of learning and teaching mathematics, 2, 59-104.

Krutetskii, V. A. (1976). The psychology of mathematical ability in schoolchildren (J. Teller, Trans.): Chicago, IL: University of Chicago Press.

Martinez-Planell, R., \& Trigueros, M. (2012). Students' understanding of the general notion of a function of two variables. Educational Studies in Mathematics, 81(3), 365-384.

Montiel, M., Vidakovic, D., \& Kabael, T. (2008). Relationship between students' understanding of functions in Cartesian and polar coordinate systems. Investigations in Mathematics Learning, 1(2), 52-70.

Montiel, M., Wilhelmi, M. R., Vidakovic, D., \& Elstak, I. (2009). Using the onto-semiotic approach to identify and analyze mathematical meaning when transiting between different coordinate systems in a multivariate context. Educational Studies in Mathematics, 72(2), 139-160.

Oehrtman, M., Carlson, M. P., \& Thompson, P. W. (2008). Foundational reasoning abilities that promote coherence in students' understandings of function. In M. P. Carlson \& C. Rasmussen (Eds.), Making the connection: Research and practice in undergraduate mathematics (pp. 150-171). Washington, DC: Mathematical Association of America.

Presmeg, N. C. (1986). Visualisation and mathematical giftedness. Educational Studies in Mathematics, 17(3), 297-311.

Presmeg, N. C. (1992). Prototypes, metaphors, metonymies and imaginative rationality in high school mathematics. Educational Studies in Mathematics, 23(6), 595-610.

Presmeg, N. C. (2006). Research on visualization in learning and teaching mathematics. Handbook of research on the psychology of mathematics education, 205-235.

Saldanha, L., \& Thompson, P. W. (1998). Re-thinking co-variation from a quantitative perspective: Simultaneous continuous variation. In S. B. Berensah, K. R. Dawkings, M. Blanton, W. N. Coulombe, J. Kolb, K. Norwood \& L. Stiff (Eds.), Proceedings of the Twenieth Annual Meeting of the North American Chapter of the International Group for the Psychology of Mathematics Education (Vol. 1, pp. 298-303). Columbus, OH: ERIC Clearninghouse for Science, Mathematics, and Environmental Education.

Smith III, J., \& Thompson, P. W. (2008). Quantitative reasoning and the development of algebraic reasoning. In J. J. Kaput \& M. Blanton (Eds.), Algebra in the Early Grades (pp. 95-132). New York, NY: Lawrence Erlbaum Associates.

Steffe, L. P., \& Thompson, P. W. (2000). Teaching experiment methodology: Underlying principles and essential elements. In R. Lesh \& A. E. Kelly (Eds.), Research design in mathematics and science education. Mahwah, NJ: Lawrence Erlbaum Associates.

Strauss, A. L., \& Corbin, J. (1998). Basics of qualitative research: Techniques and procedures for developing grounded theory (2nd ed.). Thousand Oaks, CA: Sage. 
Thompson, P. W. (1994). The development of the concept of speed and its relationship to concepts of rate. In G. Harel \& J. Confrey (Eds.), The development of multiplicative reasoning in the learning of mathematics (pp. 179-234). Albany, NY: SUNY Press.

Thompson, P. W. (2008). Conceptual analysis of mathematical ideas: Some spadework at the foundation of mathematics education. In O. Figueras, J. L. Cortina, S. Alatorre, T. Rojano $\&$ A. Sepulveda (Eds.), Proceedings of the Annual Meeting of the International Group for the Psychology of Mathematics Education (Vol. 4, pp. 45-64). Morelia, Mexico: PME.

Thompson, P. W. (2011). Quantitative reasoning and mathematical modeling. In L. L. Hatfield, S. Chamberlain \& S. Belbase (Eds.), New perspectives and directions for collaborative research in mathematics education (pp. 33-57). Laramie, WY: University of Wyoming.

Thompson, P. W. (2013). In the absence of meaning. In K. Leatham (Ed.), Vital directions for research in mathematics education (pp. 57-93). New York: Springer.

Trigueros, M., \& Martinez-Planell, R. (2010). Geometrical representations in the learning of two-variable functions. Educational Studies in Mathematics, 73, 3-19.

Weber, E., \& Dorko, A. (in press). Students' and experts schemes for rate of change and its representations. Journal of Mathematical Behavior. doi: 10.1016/j.jmathb.2014.01.002

Yerushalmy, M. (1997). Designing representations: Reasoning about functions of two variables. Journal for Research in Mathematics Education, 28(4), 431-466. 